\title{
The Electron Temperature and Anisotropy in the Solar Wind. I. Comparison of the Core and Halo Populations
}

\author{
V. Pierrard ${ }^{1,2}$ - M. Lazar $^{3,4}$. S. Poedts ${ }^{4}$ \\ . ¿̌ S. Štverák ${ }^{5,6}$. M. Maksimovic ${ }^{7}$. P.M. \\ Trávníček ${ }^{5,6,8}$ \\ Received ; accepted \\ (C) Springer $\bullet \bullet \bullet \bullet$
}

\begin{abstract}
Estimating the temperature of the solar wind particles and their anisotropies is particularly important for understanding the origin of these deviations from thermal equilibrium as well as their effects. In the absence of energetic events the velocity distribution of electrons reveal a dual structure with a thermal (Maxwellian) core and a suprathermal (Kappa) halo. This paper presents a detailed observational analysis of these two components, providing estimations of their temperatures and temperature anisotropies and decoding any potential interdependence that their properties may indicate. The data set used in this study includes more than 120000 the events detected by three missions in the ecliptic within an extended range of heliocentric distances from 0.3 to over $4 \mathrm{AU}$. The anti-correlation found for the core and halo temperatures is consistent with the radial evolution of the Kappa model, clarifying an apparent contradiction in previous observational analysis and providing valuable clues about the temperature of the Kappa-distributed populations. However, these two components manifest
\end{abstract}

\footnotetext{
${ }^{1}$ Royal Belgian Institute for Space Aeronomy, Space Physics and Solar-Terrestiral Center of Excellence, 3 av. Circulaire, B-1180 Brussels, Belgium

${ }^{2}$ Université Catholique de Louvain (UCL), Center for Space Radiations (CSR) and Georges Lemaître Centre for Earth and Climate Research (TECLIM), Earth and Life Institute (ELI), Place Louis Pasteur 3 bte L4.03.08, B-1348

Louvain-La-Neuve, Belgium

${ }^{3}$ Institut für Theoretische Physik, Lehrstuhl IV: Weltraumund Astrophysik, Ruhr-Universität Bochum, Germany

${ }^{4}$ Center for Mathematical Plasma Astrophysics, K.U.

Leuven, Celestijnenlaan 200B, 3001 Leuven, Belgium

${ }^{5}$ Institute of Atmospheric Physics, Czech Academy of

Sciences, Prague, Czech Republic

${ }^{6}$ Astronomical Institute, Czech Academy of Sciences,

Ondrejov, Czech Republic

${ }^{7}$ LESIA and CNRS, Observatoire de Paris-Meudon, Meudon, France

${ }^{8}$ Space Science Laboratory, University of California

(e-mail: viviane.pierrard@aeronomie.be)
} 
a clear tendency to deviate from isotropy in the same direction, that seems to confirm the existence of mechanisms with similar effects on both components, e.g., the solar wind expansion, or the particle heating by the fluctuations. On the other hand, the existence of plasma states with anti-correlated anisotropies of the core and halo populations suggests a dynamic interplay of these components, mediated, most probably, by the anisotropy-driven instabilities.

Keywords: Solar wind; Electron velocity distributions; Temperature anisotropy

\section{Introduction}

Ejected by the Sun in space the solar wind is an expanding plasma comprising electrons, protons and minor ions (e.g., He, O, N). At 1 AU where it is frequently observed, this plasma is very hot $\left(T_{\text {particle }}=10^{5}-10^{6} \mathrm{~K}\right)$ and very rare at the same time $\left(n=1-10 \mathrm{~cm}^{-3}\right)$, such that it requires a kinetic (comprehensive) description based on the in-situ measurements of the particle velocity distributions of the components. Measured as particle fluxes, the velocity distributions reveal two major components, a dominant low-energy core $\left(>90 \% n_{\text {total }}\right)$ and a suprathermal halo that enhances the high-energy tails of the velocity distributions and is well described by the power-law Kappa-like distribution functions (Pierrard et al., 2001; Maksimovic et al., 2005, Pierrard and Lazar, 2010). Although the halo is present in all species of plasma particles, i.e., electrons, protons or heavier ions (Vasyliunas, 1968, Christon et al., 1989: Collier et al., 1996, Štverák et al., 2008) as a distinct component markedly different from the core, the origin of these suprathermal populations is still uncertain. Multiple plausible scenarios have been proposed, including a coronal origin (Scudder, 1992 Pierrard et al., 1999; Viñas et al., 2000) of the suprathermals, as well as their generation by heating the resonant plasma particles by the fluctuations (Ma and Summers, 1998) transported by the super-Alfvénic solar wind, or the isotropisation of the field-aligned streams (also known as strahls) by the selfgenerated instabilities (Maksimovic et al., 2005, Gary and Saito, 2007, Pagel et al., 2007; Pierrard et al., 2011; Vocks, 2012; Pavan et al., 2013). Not only the origin of these two components is still controversial, but also their interplay and subsequent implication in the solar wind dynamics remains unclear (Lin, 1998, Lazar, Schlickeiser, and Poedts, 2012).

In addition to the number density criteria mentioned above, the temperature and its anisotropy $T_{\|} \neq T_{\perp}$, in general with respect to the uniform magnetic field, can also be invoked as macroscopic parameters to separate and describe the core and halo components. The magnitude of the temperature anisotropy may provide important clues on the macro- or microscopic mechanisms at work in the solar wind. For instance, the observations do not confirm an excessive increase of the temperature of plasma particles in direction parallel to the interplanetary magnetic field as an effect of adiabatic expansion of the solar wind. The observed limits of the temperature anisotropy may be an effect of the microscopic processes, like particle-particle or wave-particle interactions, which prevent large deviations from isotropy. The observational analysis proposed in 
the present paper attempts therefore to unveil detailed features of the temperature anisotropy of the electron core and halo components, and create premises for a realistic interpretation of these physical processes.

Although the first reliable information on the temperature anisotropy of the solar wind electrons may be dated back in '70s (Serbu, 1972; Scudder et al., 1973 Feldman et al., 1975), the number of subsequent reports with systematic studies is relatively limited (Phillips et al., 1989, Phillips and Gosling, 1990 Pilipp et al., 1990, Śtverák et al., 2008). In the early studies the electron temperature and the temperature anisotropy were determined by fitting the velocity distribution measured in-situ with standard bi-Maxwellian models. The core temperatures $T_{c, \|}$ and $T_{c, \perp}$, determined by bi-Maxwellian fits in directions parallel and perpendicular to the magnetic field, respectively, decrease faster with distance from the Sun than the components of the total temperature $T_{\|}$ and $T_{\perp}$ as determined from the entire electron distributions (Pilipp et al., 1990). At higher energies, the halo electrons were examined by fitting the suprathermal tails of the distribution to a second bi-Maxwellian with a lower density but higher temperatures (Feldman et al., 1975: Phillips et al., 1989, Maksimovic et al., 2000), or to a bi-Kappa also known as a bi-Lorentzian distribution function (Maksimovic et al., 2005, Štverák et al., 2008).

Correlations with plasma stream structures indicate that the electron temperatures are generally higher in the slow solar wind than in high-speed stream (Pilipp et al., 1990). An excess of parallel temperature $T_{\|}>T_{\perp}$ has been observed to dominate the observations and it is significantly larger in highspeed streams than in the slow solar wind, while an excess of perpendicular temperature $T_{\perp}>T_{\|}$is more specific to low speed and high density conditions. The core anisotropy also varies systematically as a function of electron density, being diminished with increasing the density (Phillips et al., 1989, Phillips and Gosling, 1990). It was also established a direct interdependence between the self-collisional frequency and the anisotropy of electrons (Salem et al., 2003). The existing information about the anisotropy of suprathermal electrons is less detailed compared to the core. Limitations in this case may also result from describing the halo with bi-Maxwellian models which cannot reproduce accurately the suprathermal tails, and from the fact that all these analyses were restrained to a reduced number of events (Feldman et al., 1975; Pilipp et al., 1987, Pilipp et al., 1990; Phillips et al., 1989). However, it seems to be evident that the halo component is more anisotropic than the core, a result in agreement with the assumption that suprathermal electrons have Coulomb scattering times much longer than the core electrons, and comparable to their transit times to 1 AU. Similar to the core, the electron halo exhibits in general an excess of parallel temperature $T_{h, \|}>T_{h, \perp}$ which is enhanced with the increase of the solar wind flowing speed, most probably because of the field aligned strahl which is frequently included in the halo component.

Many of these preliminary results are re-confirmed by the more advanced studies which provide a detailed parametrization of the solar wind electrons by fitting the core component to a bi-Maxwellian, the halo to a bi-Kappa and the strahl to a drifting bi-Kappa (Maksimovic et al., 2005, Štverák et al., 2008). These studies consider a large number of events combining solar wind data from 
different spacecraft missions, e.g., Helios I and II, Ulysses, Wind, and Cluster II, and within a wide range of heliographic coordinates. More pronounced in the fast winds the field-aligned strahl is diminished with heliocentric distance, especially beyond $1 \mathrm{AU}$, while the halo component is enhanced, suggesting the existence of a scattering mechanism that isotropize the strahl population (Maksimovic et al., 2005). An enhancement of the suprathermal populations quantified by a decrease of the power-index kappa (noted $\kappa$ in the next) with radial distance from the Sun is also observed, but this result seems to be in contradiction with the same trend of decreasing found for the Kappa (halo) temperature. On the other hand, a dual model combining a bi-Maxwellian core and a bi-Kappa halo is sufficient to parameterize the anisotropic electrons not only in the slow wind, but for any fast or slow wind conditions beyond 1 AU. Štverák et al. (2008) have invoked a dual halo-core approach to build statistical histograms of the temperature anisotropy of these two components for the slow and fast wind conditions in the ecliptic. The limits of the temperature anisotropy for the core and halo populations are provided for the full range of electron plasma beta parameter, enabling comparative studies with the constraints predicted by the resulting instabilities, Coulomb collisions, or adiabatic expansion of the solar wind. However, Štverák et al. (2008) have limited to study in detail only the core anisotropy, showing that greater temperature anisotropies can develop at larger distances from the Sun, and collisions still may have an effect to maintain low levels of temperature anisotropy complementing the kinetic instabilities which constrain large deviations from isotropy of the core population.

In the present paper we report the results of a supplementary extended investigation on the same set of data sampled in Štverák et al. (2008). Our main aim is to complete the puzzle by exploring the physical features of the suprathermal halo electrons, which here are presented by contrast to the properties of the core population. A comprehensive picture of these populations and their properties is expected to provide answers to the questions that are still open as well as premises for understanding the origin of these populations and their implication in key processes like particle heating or particle and energy transport in the solar wind.

\section{Fitting models for the velocity distributions}

The data set we propose to analyze in these series of papers are the main plasma parameters which describe the electron populations, namely, number density $(n)$, temperature $(T)$, temperature anisotropy $\left(A=T_{\perp} / T_{\|}\right)$, plasma beta $\left(\beta=8 \pi n k_{B} T / B_{0}^{2}\right)$, and the power-index $\kappa$ to quantify the presence of suprathermal halo population. These parameters were estimated by fitting the observed velocity distributions with a dual core-halo (subscripts $c$ and $h$, respectively) analytical model (Maksimovic et al., 2005)

$$
f\left(v_{\|}, v_{\perp}\right)=f_{c}\left(v_{\|}, v_{\perp}\right)+f_{h}\left(v_{\|} \cdot v_{\perp}\right)
$$

Such a model may describe these two principal components even in the presence of a strahl (beaming) component, which is present especially in the fast winds 
$\left(V_{\mathrm{SW}}>400 \mathrm{~km} / \mathrm{s}\right)$. With an anti-Sunward orientation, the field-aligned strahl overlaps asymmetrically in the velocity distribution (Pilipp et al., 1987; Ogilvie et al., 2000 Stverák et al., 2008; Viñas et al., 2010) and can therefore be subtracted. In the present study we focus our attention exclusively on the core and halo populations of electrons, which are always present in the observations. Moreover, the halo is significantly enhanced with heliographic distance while the strahl is diminished in the same measure (Maksimovic et al., 2005).

Both the core and halo components can be anisotropic with respect to the magnetic field direction, but their distributions are assumed gyrotropic and well approximated in polar coordinates $\left(v_{x}, v_{y}, v_{z}\right)=\left(v_{\perp} \cos \phi, v_{\perp} \sin \phi, v_{\|}\right)$by twoaxis distribution functions. Thus, the core is described by a bi-Maxwellian

$$
f_{c}\left(v_{\|}, v_{\perp}\right)=\left(\frac{m}{2 \pi k_{B}}\right)^{3 / 2} \frac{n_{c}}{T_{c, \|}^{1 / 2} T_{c, \perp}} \exp \left[-\frac{m}{2 k_{B}}\left(\frac{v_{\|}^{2}}{T_{c, \|}}+\frac{v_{\perp}^{2}}{T_{c, \perp}}\right)\right]
$$

while the halo is best fitted by a bi-Kappa

$$
\begin{aligned}
f_{h}\left(v_{\|}, v_{\perp}\right)= & \left(\frac{m}{(2 \kappa-3) \pi k_{B}}\right)^{3 / 2} \frac{n_{h}}{T_{h, \|}^{1 / 2} T_{h, \perp}} \frac{\Gamma[\kappa+1]}{\Gamma[\kappa-1 / 2]} \\
& \times\left[1+\frac{m}{(2 \kappa-3) k_{B}}\left(\frac{v_{\|}^{2}}{T_{h, \|}}+\frac{v_{\perp}^{2}}{T_{h, \perp}^{2}}\right)\right]^{-\kappa-1} .
\end{aligned}
$$

Fitting parameters are the number densities of these two components, $n_{c}$ and $n_{h}$, and the temperature components as moments of second order

$$
\begin{aligned}
& T_{c, \|}=\frac{m}{n_{c} k_{B}} \int d \mathbf{v} v_{\|}^{2} f_{c}, \quad T_{c, \perp}=\frac{m}{2 n_{c} k_{B}} \int d \mathbf{v} v_{\perp}^{2} f_{c}, \\
& T_{h, \|}=\frac{m}{n_{h} k_{B}} \int d \mathbf{v} v_{\|}^{2} f_{h}, \quad T_{h, \perp}=\frac{m}{2 n_{h} k_{B}} \int d \mathbf{v} v_{\perp}^{2} f_{h}
\end{aligned}
$$

In the limit of a very large power-index $\kappa \rightarrow \infty$ the bi-Kappa reduces to a bi-Maxwellian, leading to a standard description for both the core and halo components, see the two-Maxwellian model in Feldman et al. (1975) or Maksimovic et al. (1997). These details on the velocity distribution functions will help understanding the results of our study in the next, but first we reintroduce the observational data and discuss their implications.

\section{Observational data}

Štverák et al. (2008) have fitted the electron velocity distributions for, roughly, 124,000 events at low latitudes (in the ecliptic). These events were collected by three spacecraft as follows, see also Table I in Štverák et al. (2008): (1) $\sim 100,000$ samples from Helios 1, in the time interval 1975 - 1978, and small heliocentric distances $0.3-1.0 \mathrm{AU} ;(2) \sim 10,000$ samples from Cluster II, in a 
two-years interval 2002 - 2003, at 1 AU; and (3) 14, 000 samples from Ulysses in another two-years interval 1990 - 1991, and large distances 1.2 - 3.95 AU. Detailed descriptions for the electron analyzers used by these three missions, and for the methods of correction and reconstruction of the full $3 \mathrm{D}$ electron velocity distribution functions are provided in Štverák et al. (2008). In the presence of strahl the observed distributions are asymmetric but only data points measured in the sunward direction were fitted to the model (1), assuming that both the core and halo populations have the same properties in the antisunward direction. Data below the one count level were removed. The other plasma parameters invoked in our study, e.g., solar wind bulk speed, heliocentric distance, and the ambient magnetic field, are taken from measurements made by the plasma instruments on board these three spacecraft.

This data set has been used for studying the observed limits of the electron temperature anisotropy for the core and halo populations (Štverák et al., 2008), in an endeavor to explain their physical constraints. These limits were compared with the thresholds of the temperature anisotropy driven instabilities: For the Maxwellian core in the slow wind the limits of the temperature anisotropy appear to be well shaped by the instability thresholds predicted for the anisotropic biMaxwellian distributions, see top panel in Fig. 5 from Štverák et al. (2008). However, the same instability thresholds derived for bi-Maxwellian distributed plasmas are not relevant for the halo component, see Fig. 6 in Štverák et al. (2008), because the main quantities describing the halo populations (i.e., particle number density, temperatures, anisotropy) are determined by fitting their distributions with anisotropic bi-Kappa models, which reproduce a bi-Maxwellian only in the limit of a very large power-index $\kappa \rightarrow \infty$ (i.e., in the absence of suprathermal populations).

The same analytical model in Eq. (1) has been used by Maksimovic et al. (2005) for a different set of data combining events from the ecliptic detected by Helios 1 and 2 (1976) and Wind (1995), and events reported by Ulysses (1995) from time intervals corresponding to the first south and north polar passes. They present a radial evolution of the electron plasma components from 0.3 to 1.5 AU, building radial profiles for their main parameters, e.g., temperatures for the core $\left(T_{c}\right)$ and halo $\left(T_{h}\right)$ populations, and number densities for any of the core $\left(n_{c}\right)$, halo $\left(n_{h}\right)$ and strahl $\left(n_{s}\right)$ components (the strahl density is obtained by subtracting the core and halo electrons from total distribution). It was thus found that the core fractional density remains roughly constant with heliocentric distance, while the halo and strahl fractional densities vary in an opposite way: the relative number of halo electrons is increasing on an apparent expense of the strahl electrons which decrease with radial distance. Moreover, the radial profile of the power-index $\kappa$ indicates a decrease from about $\kappa=6-7$ at $0.3-$ 0.4 AU to $\kappa \gtrsim 3$ at $1.35-1.5$ AU (Štverák et al., 2009). These are the first important evidences confirming the scenario that the heliospheric electron halo population consists partly of electrons that have been scattered out of the strahl, also suggesting that kinetic instabilities induced by the strahl (e.g., electrostatic or electromagnetic beam-plasma instabilities) may be a source of pitch-angle scattering dominating the magnetic focusing of the strahl and enhancing the halo population (Pagel et al., 2007, Gary and Saito, 2007, Vocks, 2012). 

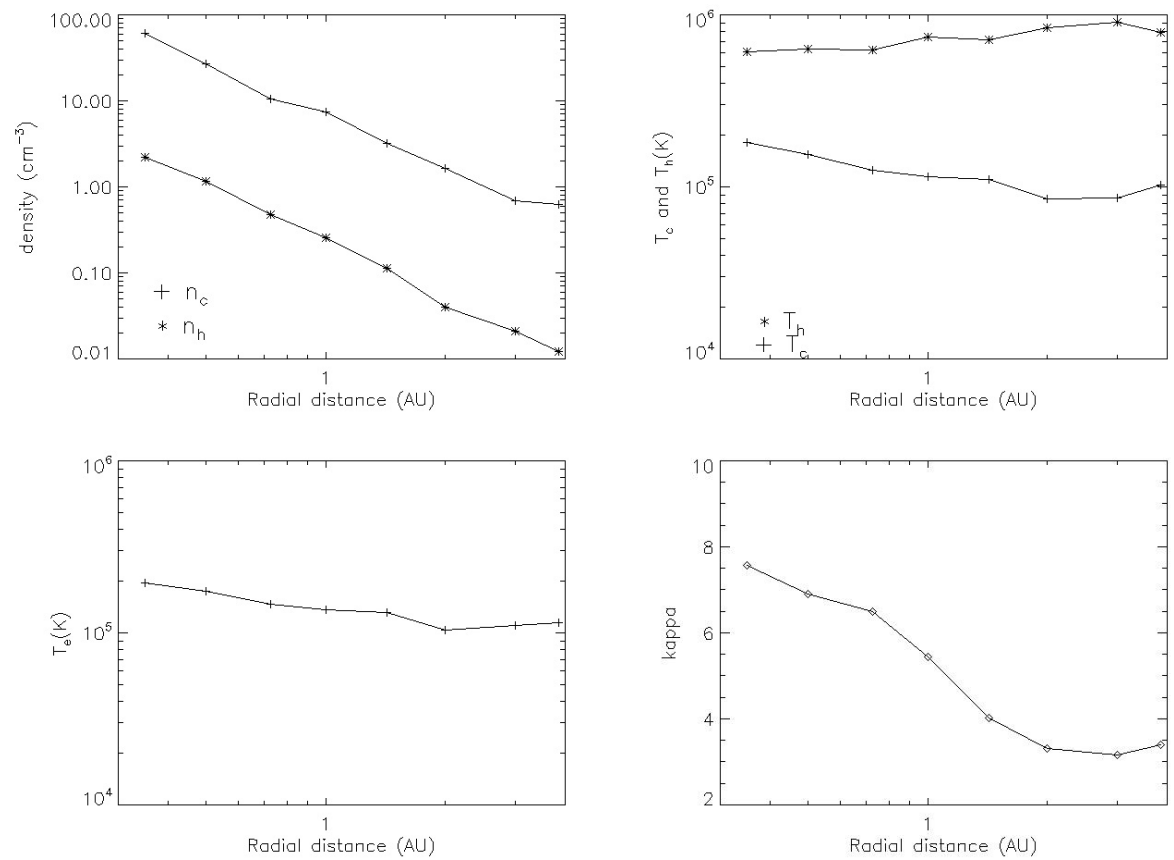

Figure 1. Radial profile of the electron parameters in the core (cross) and halo (star): (a) Electron number densities, $n_{c}$ and $n_{h}$, (b) total temperature $T_{e}=\left(n_{c} T_{c}+n_{h} T_{h}\right) /\left(n_{c}+n_{h}\right)$, (c) temperatures, $T_{c}=\left(2 T_{c, \perp}+T_{c, \|}\right) / 3$ and $T_{h}=\left(2 T_{h, \perp}+T_{h, \|}\right) / 3$, and (d) the power-index $\kappa$ for the halo component.

\section{Comparative analysis: halo vs. core}

Here we present the results of our comparative analysis on the main properties of the electron core and halo populations distinctively provided by the moments of the velocity distributions measured at low latitudes in the solar wind. Mean values of the plasma parameters used in our analysis are listed in Tables 1 and 2. For the radial evolution (Table 1) the average values are calculated for 8 bins scalled in AU units: 0.3-0.4; 0.475-0.53; 0.7-0.75; 0.9-1.1; 1.35-1.5; 1.9-2.1; 2.9-3.1; 3.75-3.95, where the first five radial bins are chosen as in Maksimovic et al. (2005). For a $\kappa$-dependency (Table 2 ) the average values are determined for 13 bins: $<2.75 ; 2.75-3.25 ; 3.25-3.75 ; 3.75-4.25 ; 4.25-4.75 ; 4.75-5.25 ; 5.25-5.75$; $5.75-6.25 ; 6.25-6.75 ; 6.75-7.25 ; 7.25-7.75 ; 7.75-8.25 ;>8.25$.

The events invoked by Maksimovic et al. (2005) were restricted to heliocentric distances between 0.3 and 1.5 AU, and to the fast wind steady states, selecting only data for which the solar wind bulk speed is larger than $650 \mathrm{~km} / \mathrm{s}$. The extended data set analyzed here includes the events detected by three probes, i.e., Helios, Cluster II and Ulysses, on an interval covering a radial distance of $0.3-4 \mathrm{AU}$ and without any restriction to fast or slow wind events.

Fig. 1 displays the radial profiles of densities, temperatures and the powerindex $\kappa$, which are computed distinctively for the core (cross) and halo (star) 
Table 1. Mean values for the plasma parameters determined in the solar wind, and used to build their heliocentric radial profiles in Figs. 1 - 4 The intervals (bins) adopted in the calculation are the following (in $\mathrm{AU}$ ): 0.3-0.4; 0.475-0.53; 0.7-0.75; $0.9-1.1 ; 1.35-1.5 ; 1.9-2.1 ; 2.9-3.1 ; 3.75-3.95$.

\begin{tabular}{|c|c|c|c|c|c|c|c|c|}
\hline Distance (AU) & 0.35 & 0.5 & 0.725 & 1.0 & 1.425 & 2.0 & 3.0 & 3.85 \\
\hline$n_{c}\left(\mathrm{~cm}^{-3}\right)$ & 61.14 & 26.60 & 10.51 & 7.34 & 3.22 & 1.64 & 0.69 & 0.42 \\
\hline$n_{h}$ & 2.19 & 1.16 & 0.48 & 0.26 & 0.11 & 0.04 & 0.02 & 0.01 \\
\hline$T\left(10^{5} \mathrm{~K}\right)$ & 1.962 & 1.749 & 1.463 & 1.360 & 1.307 & 1.033 & 1.105 & 1.170 \\
\hline$\kappa$ & 7.57 & 6.89 & 6.49 & 5.43 & 4.02 & 3.31 & 3.16 & 3.40 \\
\hline$u(\mathrm{~km} / \mathrm{s})$ & 440 & 467 & 489 & 464 & 384 & 380 & 399 & 430 \\
\hline$T_{c}\left(10^{5} \mathrm{~K}\right)$ & 1.814 & 1.550 & 1.246 & 1.145 & 1.105 & 0.852 & 0.863 & 1.020 \\
\hline$T_{c, \|}\left(10^{5} \mathrm{~K}\right)$ & 1.944 & 1.702 & 1.348 & 1.259 & 1.186 & 0.861 & 0.909 & 1.083 \\
\hline$T_{c, \perp}\left(10^{5} \mathrm{~K}\right)$ & 1.749 & 1.474 & 1.194 & 1.088 & 1.064 & 0.848 & 0.839 & 0.956 \\
\hline$A_{c}(\kappa=3)$ & - & 0.86 & 0.74 & 0.89 & 0.87 & 0.99 & 0.87 & 0.87 \\
\hline$A_{c}(\kappa=5)$ & 0.80 & 0.76 & 0.83 & 0.88 & 1.01 & 0.99 & 1.02 & 1.14 \\
\hline$A_{c}(\kappa=7)$ & 0.87 & 0.87 & 0.91 & 0.90 & - & - & - & - \\
\hline$T_{h}\left(10^{5} \mathrm{~K}\right)$ & 6.092 & 6.313 & 6.243 & 7.467 & 7.148 & 8.388 & 9.070 & 7.857 \\
\hline$T_{h, \|}\left(10^{5} \mathrm{~K}\right)$ & 6.375 & 6.581 & 6.421 & 7.618 & 7.927 & 8.391 & 8.830 & 8.113 \\
\hline$T_{h, \perp}\left(10^{5} \mathrm{~K}\right)$ & 5.951 & 6.179 & 6.154 & 7.391 & 6.759 & 8.386 & 9.190 & 7.960 \\
\hline$A_{h}(\kappa=3)$ & - & 1.10 & 0.84 & 0.96 & 1.02 & 1.02 & 0.97 & 0.95 \\
\hline$A_{h}(\kappa=5)$ & 0.78 & 0.85 & 0.92 & 0.99 & 0.92 & 0.99 & 1.03 & 1.11 \\
\hline$A_{h}(\kappa=7)$ & 0.93 & 0.97 & 1.01 & 1.00 & 0.94 & - & - & - \\
\hline
\end{tabular}

components. This enables direct comparison with the results in Maksimovic et al. (2005), e.g., Fig. 4 therein. We have found that the electron densities of the core and halo populations exhibit the same systematic decrease with heliocentric distance, but, contrary to the results in Maksimovic et al. (2005) the temperatures of these two components show an opposite evolution with radial distance. While the core temperature decreases and show a tendency of stabilization after $2 \mathrm{AU}$ (when probably the expansion is considerably slowed down and so is its influence on the temperature), the halo temperature increases with an apparent saturation of about $3 \mathrm{AU}$ (radial bin 7). After 3 AU the halo temperature starts to decrease but the data from larger distances after $4 \mathrm{AU}$ are not available to confirm this tendency. The decreasing gradient found for the core temperature is similar to those reported for the electron temperature in general in the ecliptic and at higher latitudes, see Issautier et al. (1998), Maksimovic et al. (2005) or Maksimovic et al. (2000) and Table 1 therein. 
Table 2. Mean values for the solar wind speed (displayed in Fig. 2), and the parallel and perpendicular temperatures (displayed in Fig. 3 as determined for each of the core and halo components (same units as in Table 1). The intervals for the $\kappa$-index values (bins) adopted in the calculation are the following: $<2.75$ $2.75-3.25 ; 3.25-3.75 ; 3.75-4.25 ; 4.25-4.75 ; 4.75-5.25 ; 5.25-5.75 ; 5.75-6.25 ; 6.25-6.75 ; 6.75-7.25 ; 7.25-7.75$ $7.75-8.25 ;>8.25$

\begin{tabular}{cccccccccccccc}
\hline$\kappa$ & 2.5 & 3. & 3.5 & 4. & 4.5 & 5. & 5.5 & 6. & 6.5 & 7. & 7.5 & 8. & 8.5 \\
\hline$u$ & 479 & 434 & 442 & 460 & 477 & 482 & 468 & 456 & 449 & 438 & 419 & 395 & 373 \\
\hline$T_{c, \|}$ & 1.07 & 1.18 & 1.26 & 1.35 & 1.35 & 1.33 & 1.32 & 1.32 & 1.37 & 1.47 & 1.65 & 1.81 & 1.87 \\
\hline$T_{c, \perp}$ & 0.85 & 1.02 & 1.08 & 1.13 & 1.12 & 1.12 & 1.13 & 1.15 & 1.21 & 1.32 & 1.52 & 1.72 & 1.80 \\
\hline$T_{h, \|}$ & 13.7 & 11.7 & 10.1 & 9.32 & 8.49 & 7.64 & 7.04 & 6.57 & 6.24 & 6.10 & 6.06 & 6.02 & 6.04 \\
\hline$T_{h, \perp}$ & 13.1 & 11.2 & 9.58 & 8.54 & 7.69 & 7.08 & 6.63 & 6.24 & 6.04 & 6.00 & 5.99 & 5.99 & 6.06 \\
\hline
\end{tabular}

For the halo temperature we have found a slow trend of increasing with heliocentric distance which was not reported before, and is opposite to the gradients of decreasing obtained by the previous studies mentioned above. However, in all these studies, excepting Maksimovic et al. (2005), the halo component was neglected or described only partially by a standard Maxwellian. In Maksimovic et al. (2005) the halo is accurately described by a bi-Kappa distribution function, and the halo temperature is found to have a nonmonotonic evolution with heliocentric distance, which is presented in their Fig. 4b. In that figure, a similar slow trend of increasing of the halo temperature with heliocentric distance can be observed for a shorter range of the ecliptic data: If we exclude the 5th (last) bin (1.35-1.5 AU) which corresponds to the Ulysses data from very high latitudes, the increase of the halo temperature becomes evident between the radial bins 2 (0.475-0.53 AU) and 4 (1 AU). Otherwise, if we consider the 5th bin, the resulting overall evolution of the halo temperature will indicate a decreasing with heliocentric distance, and this was the conclusion reached by Maksimovic et al. (2005). However, the 5th bin represents in their case the high latitudes data that may be less relevant for the ecliptic.
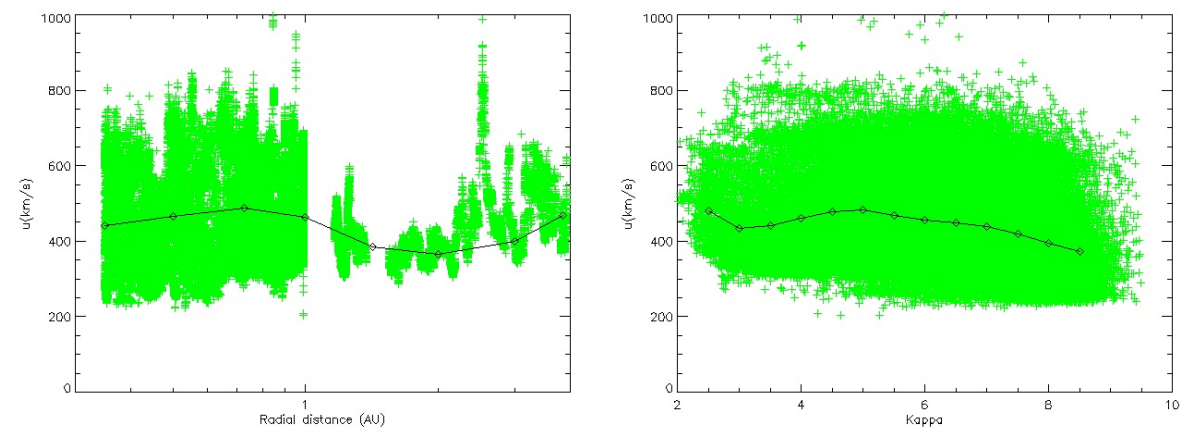

Figure 2. The solar wind flowing speed as a radial profile (left) and a function of $\kappa$ (right). 
Corresponding to the increase of the halo temperature, the power-index $\kappa$ is diminished with heliocentric distance from an average value between 7 and 8 at $0.3 \mathrm{AU}$ to about $\kappa \simeq 3$ at $3 \mathrm{AU}$. The power-index $\kappa$ from the data set in Maksimovic et al. (2005) shows the same evolution, with mention that in their study the radial bin 5 provides information from very high latitudes. Both evolutions given by the decrease of $\kappa$-index and the increase of the halo temperature are in agreement with the enhance of halo populations with radial distance concluded by Maksimovic et al. (2005).

In addition, in Fig. 2 we display both the radial profile (left panel) and the $\kappa$-dependency (right panel) of the solar wind flowing speed, which show a nonmonotonic variation. After the acceleration trend up to $0.7 \mathrm{AU}$, the solar wind speed decreases until it shows pronounced peaks at large distances $>2 \mathrm{AU}$, most probably caused by some energetic events like coronal mass ejections. To keep this observational evidence, in Fig. 2 the average values are superimposed over the scatter plot data. The right panel in the same figure confirms a tendency of increasing of the power-index $\kappa$ in the slow solar winds, as observed by (Pierrard et al., 2001). In our case $\kappa$ increases to values exceeding 9, when the Kappa distribution function approaches the Maxwellian limit, see the two-Maxwellian models used by Maksimovic et al. (2000).

Fig. 3 displays the temperature components, parallel (top panels) and perpendicular (bottom panels) to the magnetic field direction, for both the core (left panels) and halo populations (right panels). The core show a modest increase of both the temperature components with the $\kappa$-index, while the halo seems to compensate, exhibiting a very clear decrease of the parallel and perpendicular temperatures with increasing $\kappa$. These evolutions of the halo temperatures appear to be in perfect agreement with the recent theoretical studies (Lazar et al., 2015b), which indicate an increase of the Kappa temperature with a decrease of $\kappa$, i.e., an enhance of suprathermal populations, rather than a $\kappa$-independent temperature (Livadiotis and McComas, 2013). An increase of $T_{h}$ with decreasing $\kappa$ is also in accord with the radial profiles of these two quantities provided by our analysis above. Moreover, such a $\kappa$-dependence of the core and halo temperatures clarifies an apparent contradiction in Maksimovic et al. (2005), where the halo temperature is found to decrease with radial distance at the same time with an increase of the power-index $\kappa$. As discussed above, the radial decrease of $T_{h}$ found by Maksimovic et al. (2005) is given by the high-latitude data, most probably with a lower relevance for the radial evolution in the ecliptic. These comparisons indicate a decrease of $T_{h}$ at higher latitudes, but a rigorous analysis needs an extended mapping of the Ulysses data.

From a radial profile of the power-index $\kappa$ in Fig. 1, bottom-right panel, we may identify three groups of events associated to three distinct (median) values of the power-index as follows: $\kappa=7$ typical for low heliocentric distances $R<0.7 \mathrm{AU}, \kappa=5$ specific for intermediary distances $0.7 \mathrm{AU}<R<1.5 \mathrm{AU}$, and $\kappa=3$ representative for large radial distances $R>1.5$ AU. From the total number of events we select for further analysis only those associated with these three characteristic values of the power-index $\kappa=3,5$, and 7 . Thus, Fig. 4 presents a radial evolution of the temperature anisotropy for each of these three classes of events, building their radial profiles and making a comparison 

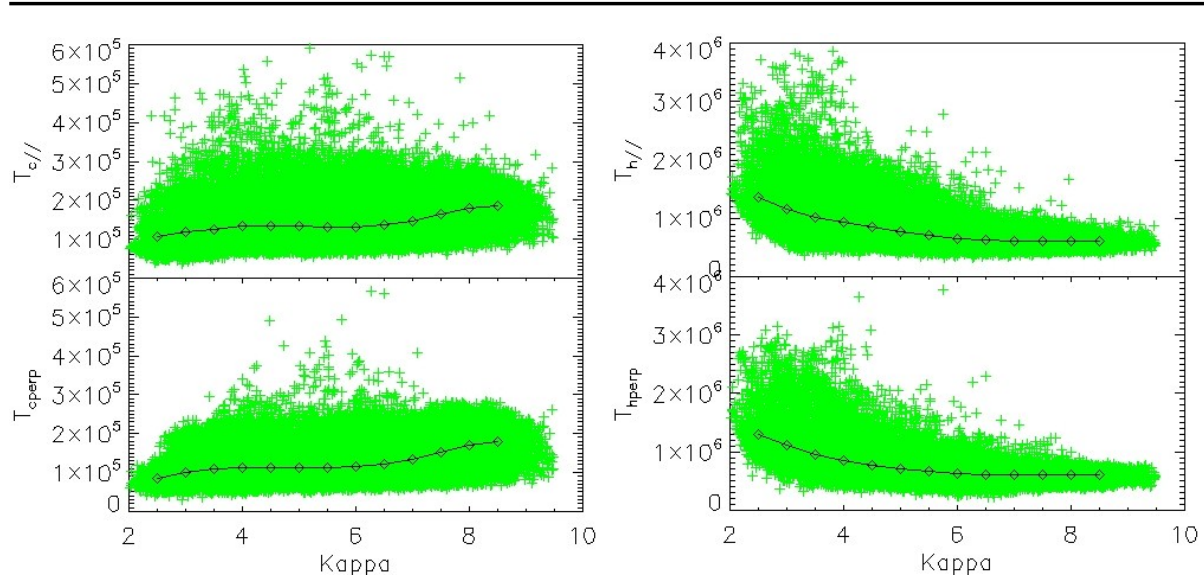

Figure 3. Temperature components parallel (top) and perpendicular (bottom) to the magnetic field vs. kappa index for the core (left) and halo (right) populations.

between the temperature anisotropy of the core and halo populations. Although these populations show deviations from isotropy in both directions, namely, $A=$ $T_{\perp} / T_{\|}>1$ due to an excess of perpendicular temperature, or $A=T_{\perp} / T_{\|}<1$ by a surplus of parallel temperature, dominant seems to be the number of states with $A<1$. The mean values of the temperature anisotropy (superimposed over the scatter data points in Fig. 4 do not deviate much from the conditions of isotropy $A_{c}=1$ and $A_{h}=1$, and have therefore a reduced relevance. Relevant in this case is the spread of data points, which is larger, sometimes markedly larger for the halo than for the core populations. Deviations from isotropy decrease with increasing $\kappa$, being thus larger for a lower $\kappa=3$ while the higher $\kappa=7$ populations are narrower and more compact.

In order to correlate the anisotropies of the core and halo populations and have a better image of their spread, in Fig. 5 we plot the halo anisotropy vs. the core anisotropy for each of these sets of events with the power-index in the range $\kappa=3 \pm 10 \% \in[2.7,3.3]$ in panel a, the events with $\kappa=5 \pm 10 \% \in[4.5,5.5]$ in panel $\mathrm{b}$, and the events with $\kappa=7 \pm 10 \% \in[6.3,7.7]$ in panel c. The data points are not scattered uniformly in the four quadrants delimited by the isotropic temperatures for the core $A_{c}=1$ and halo $A_{h}=1$, and show a dominant presence in the left quadrants defined by $A_{c}<1$, and especially in that of the left-bottom corner where both the core and halo anisotropies, i.e., $A_{c}<1$ and $A_{h}<1$, are favorable to an excess of parallel temperature. On the other hand, the data points in all these three panels show a prevailing disposition of aligning to a straight-line (linear regression) which connects the origin of axes at $A_{c}=0$ and $A_{h}=0$, with the point defined by the isotropic states for both components $A_{c}=1$ and $A_{h}=1$. The spread of data points around this line becomes more balanced with increasing $\kappa$-index. This linear regression suggests a linear correlation between the core and halo anisotropies of a form $A_{h}=a A_{c}+b$, with $a \simeq 1$ and $b \simeq 0$, by which these two components manifest a tendency to deviate from isotropy in the same direction, either the predominant states with both $A_{c}<1$ and $A_{h}<1$, or the opposite states with both $A_{c}>1$ and $A_{h}>1$. 

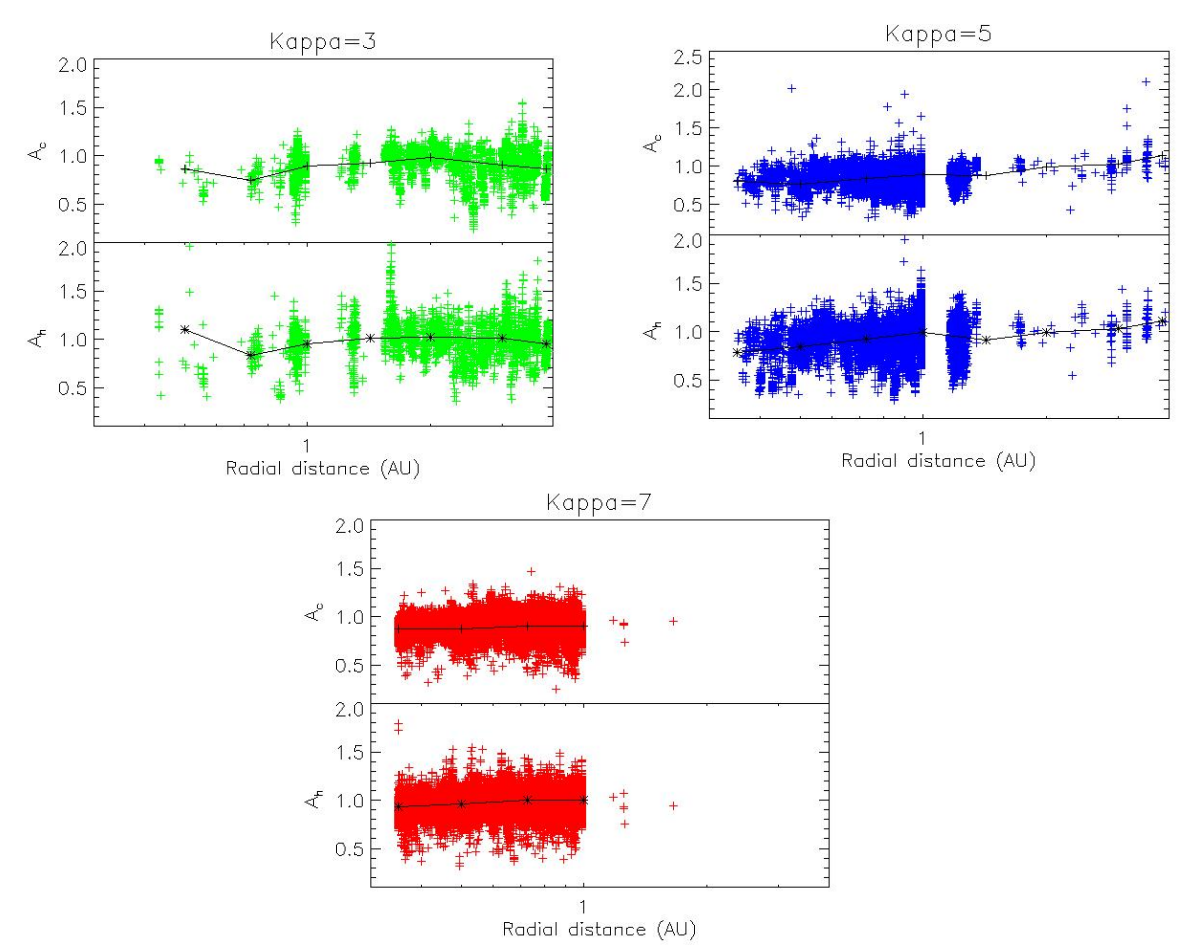

Figure 4. Radial profiles for the temperature anisotropy for three classes of events associated to three distinct values of the power index: $\kappa \simeq 3 \in[2.7,3.3]$ (top-left panel), $\kappa \simeq 5 \in[4.5,5.5]$ (top-right panel) and $\kappa \simeq 7 \in[6.3,7.7]$ (bottom panel).

Apparently, this correlation of the data points becomes less pronounced with the increase of kappa index, e.g., $\kappa=5,7$, when their tendency of aligning is reduced but still distinguishable. However, this can be only a consequence of the increase of data points for higher values of kappa index, especially for $\kappa=5$. With the increase of $\kappa$, the data points also show a concentration towards the stability states of isotropy and thermal equilibrium for both components, i.e., $A_{c}=1$ and $A_{h}=1$. This may be a natural predisposition as the increase of $\kappa$ indicates a thermalization of the halo component most probably under the effect of collisions and fluctuations, which may reduce both the suprathermal populations and the temperature anisotropy. Otherwise, noticeable is also the presence of anticorrelated states when the core and halo anisotropies are opposite, either $A_{c}>1$ and $A_{h}<1$, or $A_{c}<1$ and $A_{h}>1$. The existence of these anticorrelated states may have many implications, suggesting, for instance, different origins of the core and halo components (Scudder, 1992, Pierrard et al., 1999), but also a dynamic interplay of these populations mediated, most probably, by the kinetic instabilities (Lazar, Poedts, and Schlickeiser, 2014, Shaaban et al., 2016). 

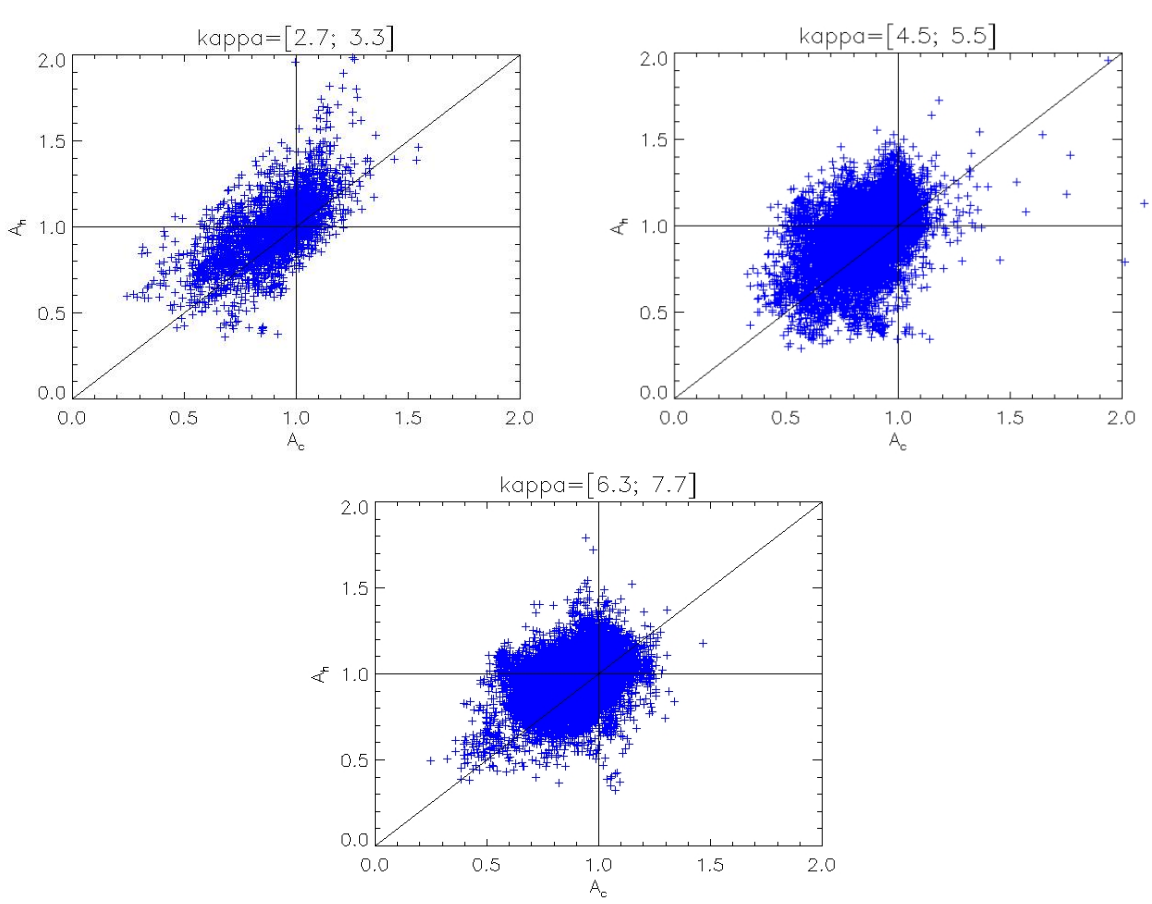

Figure 5. The electron temperature anisotropies $A_{h}$ vs. $A_{c}$ for the same classes of events chosen in Fig. 4

\section{Discussions and conclusions}

Estimating the anisotropy of the electron temperature in the solar wind has a particular importance for understanding the implications as well as the physical mechanisms which are responsible for these deviations from thermal equilibrium, e.g., the solar wind expansion, or the heating of plasma particles by the fluctuations. Large deviations from isotropy are local sources of instabilities that may enhance the fluctuations, and act in turn, pitch-angle scattering the particles and limiting the increase of temperature anisotropy. On the other hand, the solar wind is a hot and dilute plasma, where particle-particle collisions are rare and therefore expected to maintain close to the thermal equilibrium only the low-energy core populations. This is confirmed by the observations, which also show that large deviations from temperature isotropy appear to be better constrained by the resulting instabilities (Štverák et al., 2008). More intriguing are the suprathermal populations, which are less dense than the core, but hotter, such that their kinetic energy density is comparable to that of the core, i.e., $\beta_{h} \sim \beta_{c}$, see in Maksimovic et al. (2000) and Lazar et al. (2015a). In these preliminary estimations both the core and halo populations are interpreted with idealized Maxwellian models, but the results strongly point out that these two components may have similar kinetic effects, and neither of them can be ignored in the favor of the other. Decoding the interplay of the thermal core and the 
suprathermal halo seems to be vital for a correct understanding of the kinetic mechanisms in the solar wind.

In this paper we have analyzed both these two components by contrast, focusing on their temperatures and temperature anisotropies. The data set analyzed here includes more than 120000 events detected in the ecliptic from 0.3 to over $4 \mathrm{AU}$ by three spacecraft missions. According to the previous analysis (Maksimovic et al., 2005) both the core and halo temperatures are expected to decrease with heliocentric distance, but from our data set the halo temperature is found to be enhanced with increasing the distance from the Sun. This apparent contradiction can be however explained by the difference between the data sets considered in these investigations. Thus, our data set restricts to the events in the ecliptic, while the previous analysis combines data from the ecliptic with high latitude data. Without these data collected by Ulysses from over the solar poles, the halo temperature in the ecliptic also show a radial increasing similar with the one found in the present paper. On the other hand, the power-index $\kappa$ is found to be diminished with heliocentric distance, suggesting that the core temperature $T_{c}$ and the halo temperature $T_{h}$ must show opposite variations with the $\kappa$-index, i.e., a decrease of $\kappa$ should determine an increase of $T_{h}$, while $T_{c}$ is diminished in the same measure. These variations are indeed confirmed by the observations in Fig. 3 , and they come to provide an important observational support for the recent studies (Lazar et al., 2015b) which suggest that Kappa modelling of the plasma particles and their kinetic effects in the solar wind must consider a $\kappa$-dependent temperature, increasing with decreasing the $\kappa$-index.

Radial profiles of the temperature anisotropies have been built in Fig. 4 for three groups of events associated to distinct values of the power-index $\kappa=3,5,7$, where the lowest value is found representative for large distances from the Sun $R>1.5 \mathrm{AU}$, and the highest value of $\kappa$ for low distances $R<0.7 \mathrm{AU}$. Relevant is the spread of data points indicating deviations from isotropy, which in many situations are markedly larger for the halo than for the core. These deviations from isotropy decrease with increasing $\kappa$, such that the higher $\kappa=7$ populations are more compact along the isotropy condition $(A=1)$ than those modelled by lower values of $\kappa$. We have also looked for a direct interdependence between the core and halo anisotropies, building panels in Fig. 5. again for the same groups of events associated with three distinct values of the power-index $\kappa$. The core and halo components manifest a clear tendency to deviate from isotropy in the same direction, confirming the existence of mechanisms of energization or relaxation with the effects on both components, e.g., the solar wind expansion, or the particle heating by the fluctuations. Moreover, the existence of plasma states with anti-correlated anisotropies of the core and halo populations suggests a dynamic interplay of these components, mediated, most probably, by the anisotropydriven instabilities. Indeed, for the core population the large deviations from isotropy appear to be constrained by these instabilities Štverák et al. $(2008)$. For the halo populations such a study does not exist and it will make the object of our next investigations.

Acknowledgements The research leading to these results has received funding from the Scientific Federal Policy in the framework of the program Interuniversity Attraction Pole for the project P7/08 CHARM. The authors acknowledge support from the Katholieke Universiteit 
Leuven, the Ruhr-University Bochum, and Alexander von Humboldt Foundation. These results were obtained in the framework of the projects GOA/2015-014 (KU Leuven), G0A2316N (FWO-Vlaanderen), and C 90347 (ESA Prodex 9). The research leading to these results has also received funding from the European Commission's Seventh Framework Programme FP7PEOPLE- 2010-IRSES-269299 project-SOLSPANET (www.solspanet.eu). The authors further acknowledge the grant 15-17490S of the Czech Science Foundation.

\section{References}

Anderson, B.R., Skoug, R.M., Steinberg, J.T., McComas, D.J.: 2012, Variability of the solar wind suprathermal electron strahl, J. Geophys. Res. 117, A04107.

Feldman W. C., Asbridge J. R., Bame S. J., Montgomery M. D., Gary S.P.: 1975, Solar wind electrons, J. Geophys. Res. 80, 4181.

Christon, S.P., Williams, D.J., Mitchell, D.G., Frank, L.A., Huang, C.Y.: 1989, Spectral characteristics of plasma sheet ion and electron populations during undisturbed geomagnetic conditions, J. Geophys. Res. 94, 13409.

Collier M. R., Hamilton, D. C., Gloeckler, G., Bochsler, P., Sheldon, R. B.: 1996, Neon-20, Oxigen-16, and Helium-4 densities, temperatures, and suprathermal tails in the solar wind determined with WIND/MASS, Geophys. Res. Lett. 23, 1191-1194.

Gary, S.P., Saito, S.: 2007, Broadening of solar wind strahl pitch-angles by the electron/electron instability: Particle-in-cell simulations. Geophys. Res. Lett. 34, L14111.

Issautier, K., Meyer-Vernet, N., Moncuquet, M., Hoang, S.: 1998, Solar wind radial and latitudinal structure: Electron density and core temperature from Ulysses thermal noise spectroscopy. J. Geophys. Res. 103, 1969.

Lazar, M., Schlickeiser, R., Poedts, S.: 2012, Suprathermal Particle Populations in the Solar Wind and Corona, in Exploring the Solar Wind, ed. M. Lazar (InTech), Ch. 11, http://www.intechopen.com/books/exploring-the-solar-wind

Lazar, M., Poedts, S., Schlickeiser, R.: 2014, The interplay of Kappa and core populations in the solar wind: Electromagnetic electron cyclotron instability, J. Geophys. Res. Space Physics, 119, 93959406.

Lazar, M., Poedts, S., Schlickeiser, R., Dumitrache, C.: 2015a, Towards realistic parametrization of the kinetic anisotropy and the resulting instabilities in space plasmas. Electromagnetic electron-cyclotron instability in the solar wind, MNRAS 446, 3022-3033.

Lazar, M., Poedts, S., Fichtner, H.: 2015b, Destabilizing effects of the suprathermal populations in the solar wind, Astron. Astrophys. 582, A124.

Lin, R.P.: 1998, Wind observations of suprathermal electrons in interplanetary medium, Space Sci. Rev. 86, 61 .

Livadiotis, G., McComas, D. J.: 2013, Understanding Kappa Distributions: A Toolbox for Space Science and Astrophysics. Space Sci. Rev. 175, 183.

Ma, C.-yu Summers, D.: 1998. Formation of Power-law Energy Spectra in Space Plasmas by Stochastic acceleration due to whistler-mode waves, Geophys. Res. Let. 25, 4099-4102,

Maksimovic M., Pierrard V., Riley P.: 1997, Ulysses electron distributions fitted with Kappa functions, Geophys. Res. Lett., 24, 1151.

Maksimovic, M., Gary, S.P., Skoug, R.M.: 2000, Solar wind electron suprathermal strength and temperature gradients: Ulysses observations. J. Geophys. Res. 105, 18337-18350.

Maksimovic, M., Zouganelis, I., Chaufray, J.-Y., Issautier, K., Scime, E. E., Littleton, J. E., Marsch, E., McComas, D.J., Salem, C., Lin, R.P., Elliott, H.: 2005, Radial evolution of the electron distribution functions in the fast solar wind between 0.3 and 1.5 AU. J. Geophys. Res. 110, A09104.

Ogilvie, K.W., Fitzenreiter, R., Desch, M.: 2000, Electrons in the low-density solar wind. J. Geophys. Res. 105, 27,277.

Pagel, C., Gary, S.P., de Koning, C.A., Skoug, R.M., Steinberg, J.T.: 2007, Scattering of suprathermal electrons in the solar wind: ACE observations. J. Geophys. Res. 112, A04103.

Pavan, J., Viñas, A.F., Yoon, P.H., Ziebell, L.F., Gaelzer, R.: 2013, Solar wind strahl broadening by self-generated plasma waves, Astrophys. J. 769, L30

Phillips, J.L., Gosling, J.T., McComas, D.J., Bame, S.J., Gary, S.P.: 1989, Anisotropic thermal electron distributions in the solar wind. J. Geophys. Res. 94, 6563-6579.

Phillips, J.L., Gosling, J.T.: 1990, Radial evolution of solar wind thermal electron distributions due to expasion and collisions. J. Geophys. Res. 95, 4217-4228. 
Pierrard V., Lazar, M.: 2010, Kappa Distributions: Theory and Applications in Space Plasmas, Solar Phys. 267, 153.

Pierrard V., Lazar M., Schlickeiser R.: 2011. Evolution of the electron distribution function in the wave turbulence of the solar wind. Solar Phys. 269, 2, 421-438, DOI 10.1007/s11207010-9700-7.

Pierrard V., Maksimovic, M., Lemaire, J.: 1999, Electron velocity distribution function from the solar wind to the corona. J. Geophys. Res., 104, 17021-17032.

Pierrard V., Maksimovic, M., Lemaire, J.: 2001, Core, halo and strahl electrons in the solar wind. Astrophys. Space Sci. 277, 2, 195-200.

Pilipp, W.G., Miggenrieder, H., Montogomery, M.D., Mühlhäuser, K.-H., Rosenbauer, H., Schwenn, R.: 1987, Characteristics of electron velocity distribution functions in the solar wind derived from the Helios plasma experiment. J. Geophys. Res. 92, 1075.

Pilipp, W.G., Miggenrieder, H., Mühlhäuser, K.-H., Rosenbauer, H., Schwenn, R.: 1990, Largescale variations of thermal electron parameters in the solar wind between 0.3 and $1 \mathrm{AU}$. J. Geophys. Res. 95, 6305-6329.

Salem, C., Hubert, D., Lacombe, C., Bale, S.D., Mangeney, A, Larson, D.E., Lin, R.P.: 1003, Electron properties and Coulomb collisions in the solar wind at $1 \mathrm{AU}$ : Wind observations, Astrophysical J. 585, 11471157.

Scudder, J. D.; 1992, On the causes of temperature change in inhommogeneous low-density astrophysical plasmas. Astrophys. J. 398, 299

Scudder, J.D., Lind, D.L., and Ogilvie, K.W.: 1973, Electron observations in the solar wind and magnetosheath, J. Geophys. Res. 78, 6535-6548.

Serbu, G.P.: 1972, Explorer 35 observations of solar wind electron den- sity, temperature, and anisotropy, J. Geophys. Res., 77, 1703,

Shaaban, S.M., Lazar, M., Poedts, S., Elhanbaly, A.: 2016, The interplay of the solar wind proton core and halo populations: EMIC instability, J. Geophys. Res. Space Physics, submitted.

Štverák, Š., Trávníček, P., Maksimovic, M., Marsch, E., Fazakerley, A.N., Scime, E.E.: 2008, Electron temperature anisotropy constraints in the solar wind. J. Geophys. Res. 113, A03103.

Štverák, Š., Maksimovic, M., Trávníček, P., Marsch, E., Fazakerley, A.N., Scime, E.E.: 2009, Radial evolution of nonthermal electron populations in the low-latitude solar wind: Helios, Cluster, and Ulysses Observations. J. Geophys. Res. 114, A05104.

Vasyliunas, V. M.: 1968, A survey of low-energy electrons in the evening sector of the magnetosphere with OGO 1 and OGO 3, J. Geophys. Res. 73, 2839

Viñas, A. F., Wong, H. F., Klimas, A. J.: 2000, Generation of electron suprathermal tails in the upper solar atmosphere: Implications for coronal heating, Astrophys. J. 528, 509-523

Viñas, A. F., Gurgiolo, C., Nieves-Chinchilla, T., Gary, S. P., Goldstein, M. L.: 2010, WhistlerWaves Driven by Anisotropic Strahl Velocity Distributions. AIP Conference Proceedings 1216, 265-270

Vocks, C.: 2012, Kinetic Models for WhistlerWave Scattering of Electrons in the Solar Corona and Wind. Space Sci. Rev. 172, 303. (and references therein) 